\title{
Call for Special Issue Papers: Health Equity Call for Papers: Migrant and Refugee Health
}

\section{Deadline for Manuscript Submission: October 15, 2020}

Editor-in-Chief: Ana E. Núñez, MD; Drexel University School of Medicine

Dr. Ana Núñez, Editor-in-Chief of Health Equity invites you to submit a manuscript to a Special Collection on Migrant and Refugee Health.

Meeting the healthcare needs of migrants and refugees in the United States and around the world is an escalating and challenging public health problem. The health and well-being of migrants is a culmination of the migration journey, which can involve adverse conditions in their homelands, harsh conditions during travel, and inhumane treatment at borders. Both short- and long-term adverse health effects on migrants are expected, and the likelihood of generational effects has been noted.

Point of entry guidelines have historically, and until recently, been largely concerned with detecting, treating, and containing infectious diseases. The focus has now broadened to include non-communicable diseases

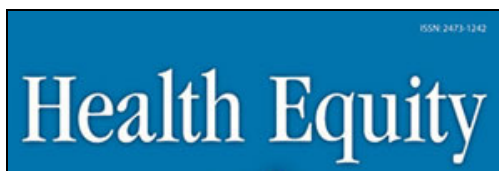
and mental health, which together are referred to as the "triple burden" of disease. Such conditions, which are preventable, treatable, and manageable, can become health inequities when the health system fails to meet the needs of any group, whether migrant or native-born.

Dr. Núñez invites you to submit papers that will examine these key topics:

- Training of the health workforce and health system to meet the legal, ethical, medical, psychosocial, cultural, linguistic and other needs of migrants, including the unique needs of women and children

- Examination of migrant needs and healthcare barriers by country of origin

- Trans-disciplinary communication to the public and policymakers by researchers, epidemiologists, academics, and other stakeholders to counterbalance misinformation and inaccurate data

- Evaluation and case-studies of community-based and grass-roots initiatives addressing migrant health needs

- Protocols, policy, and position statements by hospitals and health professional associations

- Role of foreign born and foreign trained physicians in providing care to migrants

- Case studies related to vulnerable groups, including children, women of childbearing age, and elderly persons

- Strategies to use the social determinants of health framework to identify health needs of migrants and solutions (migration also being a social determinant of health)

- Role of "cultural brokers" to help migrants access medical services and promote continuity of care 
- Studies on social and environmental factors contributing to mental health conditions in migrants (posttraumatic stress disorder, depression, anxiety, suicidal ideation, etc.)

- Education of migrant groups about disease prevention and good health practices

Submit your manuscript online at https://mc.manuscriptcentral.com/heq or email Jennifer Kuhn, jkuhn@ liebertpub.com, with any questions.

\section{Visit the Instructions for Authors: www.liebertpub.com/heq}

\section{Submit your paper for peer review online: https://mc.manuscriptcentral.com/heq}

